\title{
Single-Molecule Stochastic Resonance
}

\author{
K. Hayashi, ${ }^{1,2}$ S. de Lorenzo, ${ }^{3}$ M. Manosas, ${ }^{1,3,4}$ J. M. Huguet, ${ }^{1}$ and F. Ritort ${ }^{1,3, *}$ \\ ${ }^{1}$ Departament de Física Fonamental, Facultat de Física, Universitat de Barcelona, Diagonal 647, E-08028, Barcelona, Spain \\ ${ }^{2}$ Department of Applied Physics, School of Engineering, Tohoku University, 2-1-1 Katahira, Sendai 980-8577, Miyagi, Japan \\ ${ }^{3}$ CIBER de Bioingeniería, Biomateriales y Nanomedicina, Instituto de Salud Carlos III, Madrid, Spain \\ ${ }^{4}$ Laboratoire de Physique Statistique, Ecole Normal Supérieure, Unité Mixte de Recherche 8550 associée au Centre National de la \\ Recherche Scientifique et aux Universités Paris VI et VII, 75231 Paris, France
}

(Received 14 March 2012; published 24 August 2012)

\begin{abstract}
Stochastic resonance (SR) is a well-known phenomenon in dynamical systems. It consists of the amplification and optimization of the response of a system assisted by stochastic (random or probabilistic) noise. Here we carry out the first experimental study of SR in single DNA hairpins which exhibit cooperatively transitions from folded to unfolded configurations under the action of an oscillating mechanical force applied with optical tweezers. By varying the frequency of the force oscillation, we investigate the folding and unfolding kinetics of DNA hairpins in a periodically driven bistable free-energy potential. We measure several SR quantifiers under varied conditions of the experimental setup such as trap stiffness and length of the molecular handles used for single-molecule manipulation. We find that a good quantifier of the SR is the signal-to-noise ratio (SNR) of the spectral density of measured fluctuations in molecular extension of the DNA hairpins. The frequency dependence of the SNR exhibits a peak at a frequency value given by the resonance-matching condition. Finally, we carry out experiments on short hairpins that show how SR might be useful for enhancing the detection of conformational molecular transitions of low SNR.
\end{abstract}

DOI: 10.1103/PhysRevX.2.031012 Subject Areas: Biological Physics, Nonlinear Dynamics, Statistical Physics

\section{INTRODUCTION}

All nonlinear systems that exhibit stochastic (random or probabilistic) noise are susceptible to undergoing stochastic resonance (SR). When SR is triggered, the response of a system to an external forcing is amplified. SR has been studied in a large variety of systems, including climate dynamics [1,2], colloidal particles [3-5], biological systems [6-8], and quantum systems [9,10]. With the recent advent of single-molecule techniques, it is now possible to measure SR at the level of individual molecules. Biomolecules exhibit rough and complex free-energy landscapes that determine molecular folding kinetics and influence the way the molecules fold into their native structures. The use of force-spectroscopy techniques has become important practice in studies of molecular biophysics. By applying a mechanical force at both extremities of an individual linear molecule and then recording the time evolution of the molecular extension (the reaction coordinate in these experiments), one obtains information about the folding reaction. The application of forces makes it possible to disrupt the weak bonds that hold their native structure and to reach a stretched unfolded conformation. In this way, thermodynamics (e.g., the free energy of folding) and kinetics (the rates of unfolding and folding) can be determined.

\section{*fritort@gmail.com}

Published by the American Physical Society under the terms of the Creative Commons Attribution 3.0 License. Further distribution of this work must maintain attribution to the author(s) and the published article's title, journal citation, and DOI.
Although most SR studies use temperature as a tunable parameter, this is not the best choice for investigating SR effects in biological macromolecules at the singlemolecule level. Biomolecules have a strong sensitivity to temperature variations. Indeed, beyond affecting thermal and thermally assisted noise, temperature also modifies the shape of the molecular free-energy landscape. Thus, another tunable parameter such as the oscillation frequency of an applied force might be more appropriate for studying SR in biomolecules. In our experiments, SR appears as a maximum in the response of a biomolecule at a characteristic frequency (the resonance frequency). Similar to stochastic resonance in other physical systems, this maximum occurs when a characteristic time scale of the thermally driven dynamics of the molecule (e.g., its decorrelation or relaxation time) matches the half-period of the oscillation of the applied force-the so-called matching condition) [11]. The matching condition must be taken not as a strict equality but as a qualitative relationship between the two time scales [12,13]. In other words, different SR quantifiers may not give coincident resonance frequencies, especially for low-quality resonance peaks. It is important to investigate which SR quantifier is best suited for identifying SR behavior.

In this work, we use optical tweezers to investigate $S R$ in single DNA hairpins driven by oscillatory mechanical forces. The high chemical stability of DNA makes DNA hairpins excellent models for investigating SR at the single-molecule level. When an applied force oscillates around the average unfolding force, thermally activated hopping kinetics between the folded $(F)$ and unfolded (U) states synchronizes with the frequency of the external 
driving force, leading to SR. SR can be measured by recording the oscillations produced in the molecular extension relative to the magnitude of the thermal noise. Our primary aim in this work is to perform a systematic study of SR in single molecules exhibiting bistable dynamics, rather than using SR as a useful tool to determine the kinetic properties of DNA hairpins. In fact, these can be estimated by using other much less time-consuming methods (e.g., by directly analyzing hopping traces). However, we also carry out studies of SR in short hairpins that show how SR might prove useful for enhancing the detection of conformational transitions of low SNR.

The paper is organized as follows. In Sec. II, our experimental setup is explained. Our main SR results in DNA hairpins are presented in Sec. III, and the influence of the experimental conditions (i.e., dsDNA handle length and trap stiffness) is investigated in Sec. IV. We compare different SR quantifiers in Sec. V, and in Sec. VI we describe the related phenomenon of resonant activation. Finally, in Sec. VII, we purposely design short DNA sequences to increase the noise of the signal and test whether SR can still be used to identify the hopping rate. In Sec. VIII, we summarize our conclusions and discuss situations in which SR might be a useful technique.

\section{EXPERIMENTAL SETUP AND HOPPING EXPERIMENTS}

In Fig. 1(a), we show a schematic illustration of our experimental setup (left) and the DNA sequence of hairpin $\mathrm{H} 1$ that we investigated (upper right). The DNA hairpin is tethered between two short dsDNA handles (29 bp) that are linked to micron-size beads [14]. One bead is captured in the optical trap whereas the other is immobilized at the tip of a glass pipette [15]. By moving the position of the optical trap relative to the pipette, a force is exerted at the extremities of the hairpin. (a)

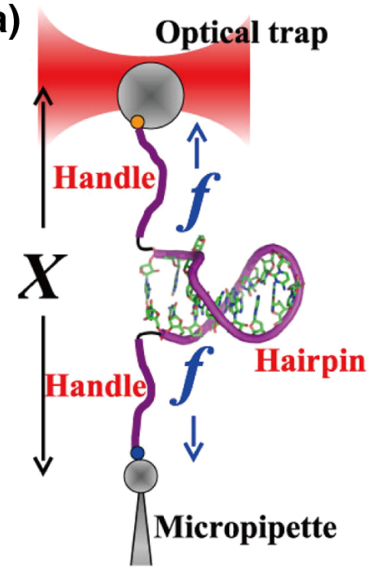

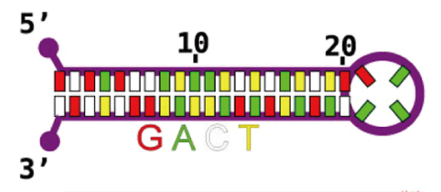

$3^{\prime}$

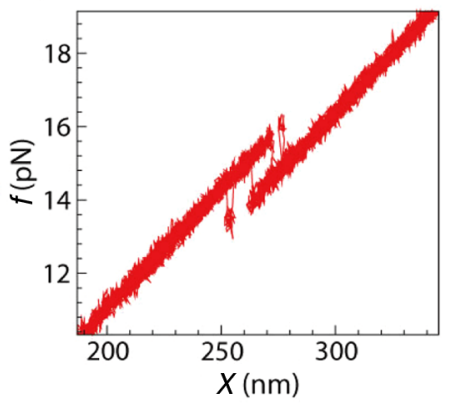

(c)
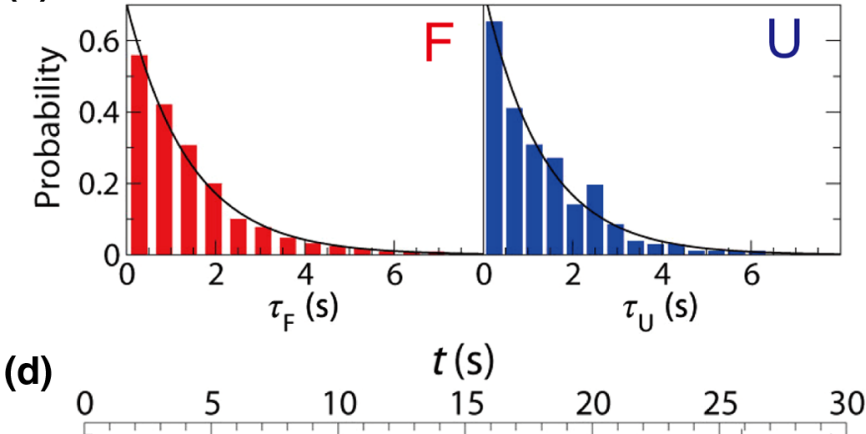

(b)
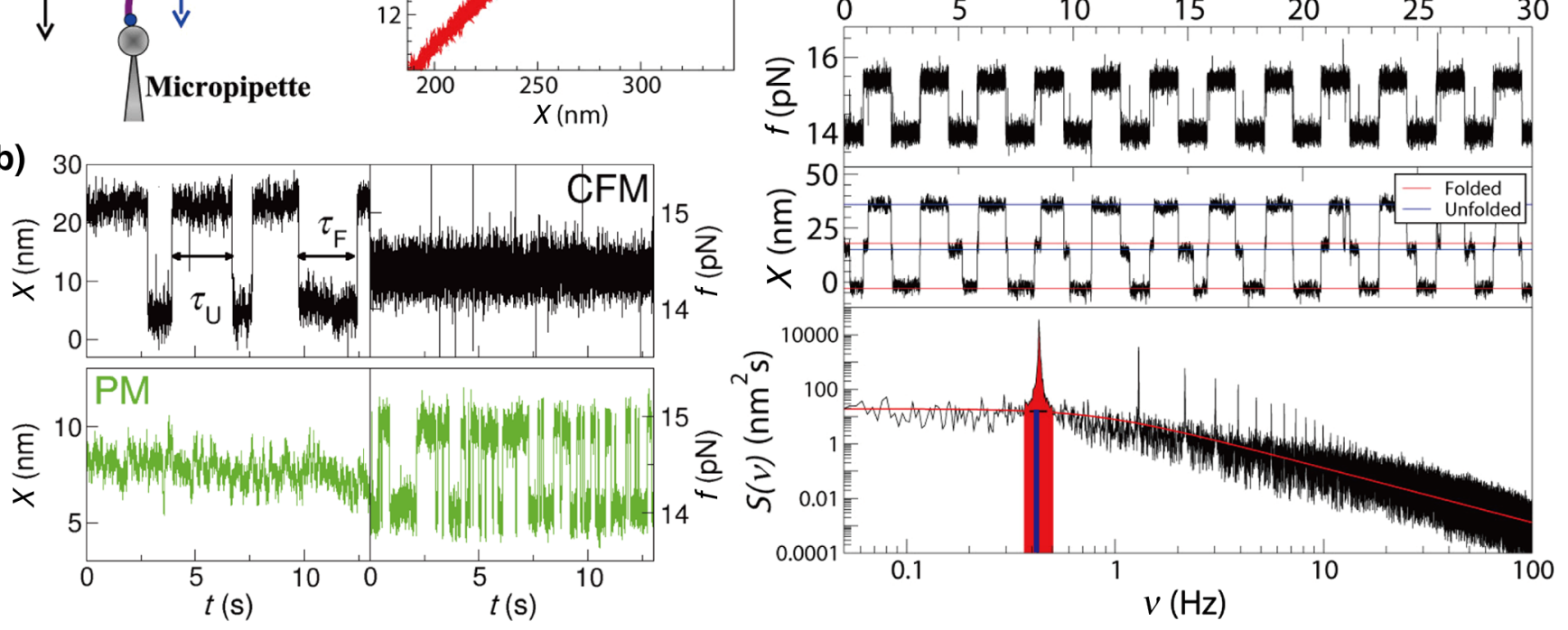

FIG. 1. Experimental setup, hopping, and SR experiments. (a) Illustration of the experimental system (left), DNA sequence of the two-state hairpin H1 (upper right, sequence shown in color code), and experimental force-distance curve for H1 obtained from a pulling experiment (lower right). (b) Typical force and extension traces of the hopping experiments for H1 obtained in CFM (upper) and PM (lower). (c) Probability distributions of the residence times for $\mathrm{H} 1$ in the F (red) and U (blue) states obtained from the hopping experiments at $f=f_{\mathrm{c}} \simeq 14.5 \mathrm{pN}$ in the CFM. The black curves show the exponential fit, $(1 / a) \exp (-\tau / a)$, to the data, with $a=1.42 \mathrm{~s}(a=1.34 \mathrm{~s})$ for the F (U) state, respectively. (d) Typical force and extension traces (upper and middle) obtained by applying an oscillating-force protocol with amplitude $A=0.7 \mathrm{pN}$ and frequency $\nu_{\mathrm{os}}=0.4 \mathrm{~Hz}$ around the coexistence force $f_{\mathrm{c}} \simeq$ $14.5 \mathrm{pN}$. In the lower panel, we show the measured power spectrum, $S(\nu)$, calculated by fast Fourier transform of $X(t)$ with window size $N=2^{17}$ shown in the middle panel. The sampling rate of the instrument is $1 \mathrm{kHz}$. The red area is the output signal [OS, Eq. (2)], and the vertical blue bar represents the background noise [BN, Eq. (3)]. 
In a pulling experiment, the optical trap is moved away from the pipette and a mechanical force is applied to the ends of the DNA construct (DNA hairpin plus DNA handles) until the value of the force at which the hairpin unfolds is reached. In the reverse process, the trap approaches the pipette and the force is relaxed until the hairpin refolds. In this experiment, the force exerted on the system, $f$, is recorded as a function of the relative trappipette distance, giving the so-called force-distance curve [Fig. 1(a), lower right]. Around a particular force, the coexistence force, $f_{\mathrm{c}} \simeq 14.5 \mathrm{pN}$, the hairpin hops between the $\mathrm{F}$ and $\mathrm{U}$ states for sufficiently low pulling speeds.

Hopping experiments can be performed in two different modes: constant-force mode (CFM) and passive mode (PM) $[16,17]$. In the CFM, the force applied to the DNA construct is maintained at a preset value by moving the optical trap through force-feedback control [Fig. 1(b), upper]. The folding and unfolding transitions of the DNA hairpin are followed by recording the trap position, $X(t)$. In contrast to the CFM, the PM is operated by leaving the position of the optical trap stationary without any feedback. The bead passively moves in the trap in response to changes in the extension of the DNA construct [Fig. 1(b), lower]. When the hairpin unfolds, the trapped bead moves toward the trap center and the force decreases; when the hairpin folds, the trapped bead is pulled away from the trap center and the force increases. The folding and unfolding transitions of the DNA hairpin are followed by recording the force, $f(t)$. In both cases (CFM and PM), the kinetic rates of hopping can be measured from the residence times of the trace $[X(t)$ in the CFM and $f(t)$ in the PM]. Figure 1(b) shows hopping traces measured in the CFM and PM at the coexistence force, $f_{\mathrm{c}} \simeq 14.5 \mathrm{pN}$, where the hairpin hops between the $\mathrm{F}$ and $\mathrm{U}$ states populating them with equal probability (i.e., the hairpin spends equal time in both states).

In this work, we have focused on the experiments at controlled force, rather than at fixed trap position. Both the hopping and the oscillation experiments (described below) are carried out using the force-feedback control because the controlled force experiments avoid undesirable drift effects in force that strongly affect the kinetics of the hairpin (see Appendix A). Therefore we have mainly carried out the experiments in the CFM by recording the position of the trap, $X(t)$. This signal exhibits dichotomic motion between the two distinct levels of extension [Fig. 1(b), upper left]. The difference between the two levels (short extension, folded; long extension, unfolded) reflects the release in extension (about $18 \mathrm{~nm}$ ) of the 44 nucleotides of hairpin H1. From $X(t)$ we can extract the residence-time distribution at each state that shows the exponential form characteristic of first-order decay processes [Fig. 1(c)]. The fit of the time distribution to an exponential function allows us to infer the average residence time. The force-dependent kinetic rates (equal to the inverse of the mean lifetimes), $k_{\mathrm{FU}}$ and $k_{\mathrm{UF}}$, are measured at the coexistence force, $f_{\mathrm{c}}=14.5 \pm 0.3 \mathrm{pN}$, giving $k_{\mathrm{c}}=k_{\mathrm{FU}}^{\mathrm{c}}=k_{\mathrm{UF}}^{\mathrm{c}} \simeq 0.66 \pm 0.04 \mathrm{~s}^{-1}$ (Table S0 in Ref. [18]).

\section{SR EXPERIMENTS}

To induce the SR phenomenon, we apply an oscillating force, $f(t)$, to the DNA hairpin using the force-feedback protocol, where $f(t)=f_{\mathrm{c}}+f_{\text {os }}(t)$. For $f_{\text {os }}(t)$ we choose a square-wave signal of amplitude $A$ and frequency $\nu_{\mathrm{os}}=$ $1 / T_{\mathrm{os}}$, where $T_{\mathrm{os}}$ is the period of oscillation [Fig. 1(d), upper]. The four distinct levels of extension observed [Fig. 1(d), middle] correspond to the molecular extensions of the hairpin in the $\mathrm{F}$ and $\mathrm{U}$ states at the two force values, $f=f_{\mathrm{c}}+A$ and $f=f_{\mathrm{c}}-A$. The power spectral density, $S(\nu)$, is defined as the Fourier transform of the stationary correlation function of the signal $X(t)$ :

$$
S(\nu)=\int_{-\infty}^{+\infty}\langle X(t) X(0)\rangle e^{-i 2 \pi \nu t} d t
$$

where $\langle\cdot\rangle$ denotes a time average over the signal. As shown in Fig. 1(d) (lower), $S(\nu)$ can be described as the superposition of a background power spectral density, $S_{N}(\nu)$, and a structure of delta spikes centered at $\nu_{n}=$ $(2 n+1) \nu_{\text {os }}(n=0,1,2, \cdots)$. In order to extract the signal from the background noise, we define the output signal (OS), the background noise (BN), and the signal-to-noise ratio (SNR) as [12],

$$
\begin{gathered}
\mathrm{OS}=\lim _{\Delta \nu \rightarrow 0} \int_{\nu_{\mathrm{os}}-\Delta \nu}^{\nu_{\mathrm{os}}+\Delta \nu} S(\nu) d \nu, \\
\mathrm{BN}=S_{N}\left(\nu_{\mathrm{os}}\right), \\
\mathrm{SNR}=\frac{\mathrm{OS}}{\mathrm{BN}}=\frac{1}{S_{N}\left(\nu_{\mathrm{os}}\right)} \lim _{\Delta \nu \rightarrow 0} \int_{\nu_{\mathrm{os}}-\Delta \nu}^{\nu_{\mathrm{os}}+\Delta \nu} S(\nu) d \nu .
\end{gathered}
$$

The SNR defined in Eq. (4) is equal to the ratio of the spectral power of the signal at the frequency $\nu_{\mathrm{os}}(\mathrm{OS})$ to the noise-floor spectral density measured in the presence of the oscillation (BN) and has dimensions of Hz. Figure 1(d) (lower) illustrates how we measure the OS (red area) and the BN (blue vertical bar) from the spectral density. Other equivalent definitions of the SNR [19] are the dimensionless ratio between the power in the output signal [Eq. (2)] and the total input power delivered by the noise [proportional to the integral of background spectral density $S_{N}(\nu)$ over all $\nu$ ]. Because the total input noise power depends only weakly on $\nu_{\text {os }}$, we can take the OS, Eq. (2), as another indicator of the SR phenomenon. Indeed, both indicators OS and SNR are equally valid for identifying resonant behavior, even though the peak is often more visible in the latter (as we discuss below) [11].

For the hairpin $\mathrm{H} 1$ at high trap power and trap stiffness $\kappa_{\text {trap }} \simeq 70 \mathrm{pN} / \mu \mathrm{m}$, the resulting OS and BN as a function of $\nu_{\text {os }}$ are depicted in Fig. 2(a) (lower), while Fig. 2(c) shows the SNR. In contrast to the OS, the presence of a peak around $\nu_{\mathrm{os}}=0.4 \pm 0.05 \mathrm{~Hz}$ is apparent for the SNR. This value is close to that predicted by the matching 
condition, $\nu_{\mathrm{SR}}=k_{\mathrm{c}} / 2$, which states that the SNR is maximum when the average hopping time of the hairpin $\left(1 / k_{\mathrm{c}}=1.56 \mathrm{~s}\right)$ is equal to half the period of the forcing oscillation $\left(1 / 2 \nu_{\mathrm{os}}=1.25 \mathrm{~s}\right)[12,20-22]$. This shows that

(a)
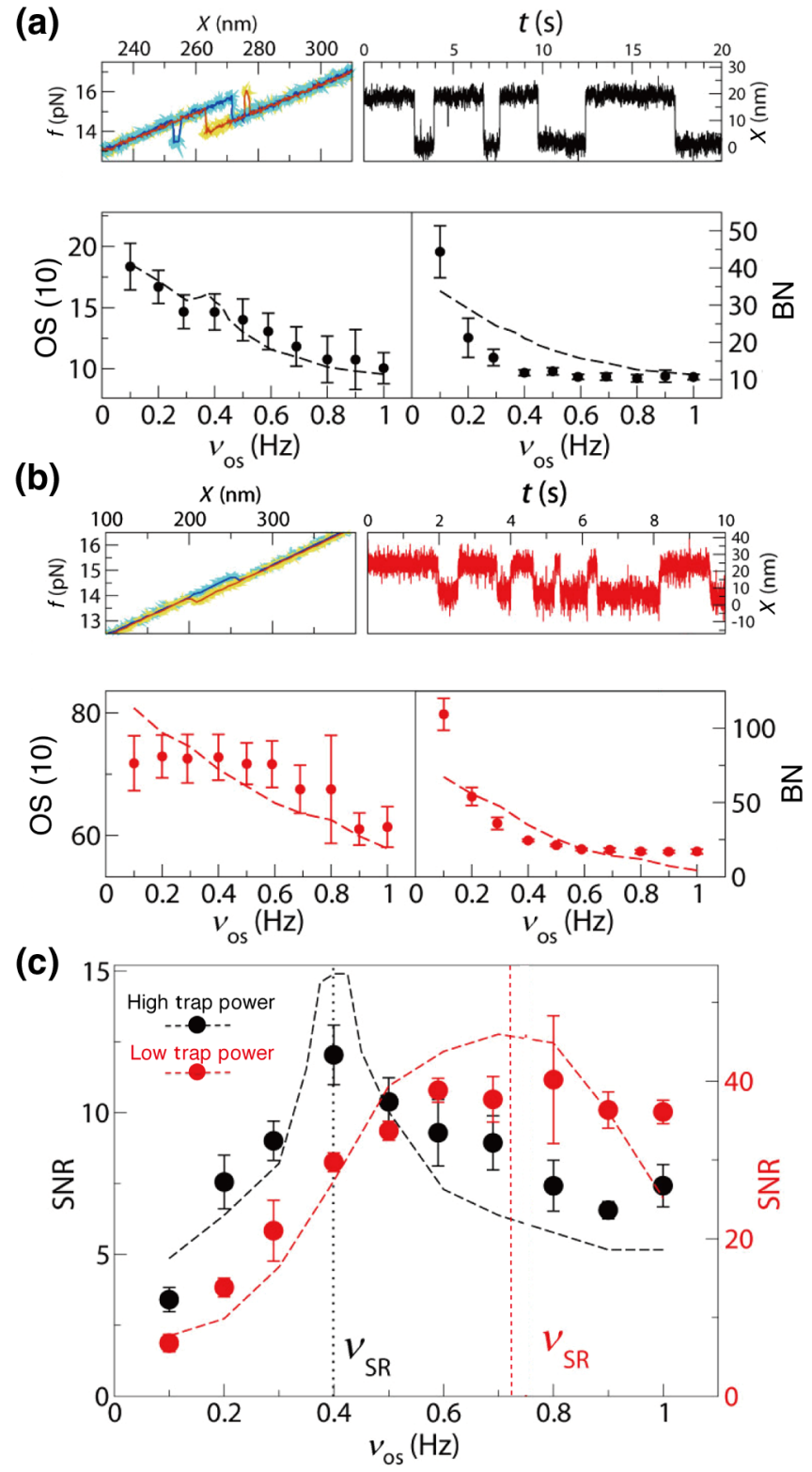

FIG. 2. SR experiments for hairpin H1 at different trap stiffness. (a) Pulling cycle (unfolding, blue; refolding, red), hopping trace, $\mathrm{OS}$, and $\mathrm{BN}$ for $\mathrm{H} 1$ with amplitude $A=0.7 \mathrm{pN}$ at high trap stiffness, $\kappa_{\text {trap }}=70 \mathrm{pN} / \mu \mathrm{m}$, and (b) the same experiments at low trap stiffness, $\kappa_{\text {trap }}=24 \mathrm{pN} / \mu \mathrm{m}$. Results have been averaged over 5-10 molecules. [Note: The force rips shown in forcedistance curves should drop vertically without any finite-stiffness correction. The finite-slope correction shown in (b) (top, left) is due to low-bandwidth filtering of data]. (c) SNR at high trap stiffness (low trap power) depicted in black (red). Units: OS $\left(\mathrm{nm}^{2}\right), \mathrm{BN}\left(\mathrm{nm}^{2} / \mathrm{Hz}\right), \mathrm{SNR}(\mathrm{Hz})$. Simulation results are shown as dashed lines (Figs. S4 and S7 in Ref. [18]). The error bars represent the standard error over different molecules.
SR in single-molecule hopping experiments approximately fulfills the matching condition as has been observed in other bistable systems.

The OS and the SNR can be calculated theoretically as a function of the oscillation frequency for a Brownian particle in a continuous double-well potential $[11,20,23]$. In this model, the OS and the SNR exhibit a soft and a sharp peak, respectively, only when SR is induced at large enough forcing amplitudes [11]. These large forcing amplitudes correspond to a nonlinear regime of the system in which the shape of the double-well potential is so deformed that the barrier separating the wells vanishes at the maximum of the oscillation. In our experiments, we apply a large oscillation amplitude $(A=0.7 \mathrm{pN})$. Note that the region of coexistence between the $\mathrm{F}$ and the $\mathrm{U}$ states spans less than $3 \mathrm{pN}$ in Fig. 1(a) (lower right). Thus an extra force of $0.7 \mathrm{pN}$ strongly alters the barrier and the relative free energy between states $\mathrm{F}$ and $\mathrm{U}$. Our experimental results agree with the theoretical predictions by Stocks [11] obtained in the nonlinear-response regime. We have performed a numerical simulation of an overdamped particle moving in a double-well potential with parameters that fit the experimentally measured molecular free-energy landscape (Sec. IV in Ref. [18]). Despite its simplicity, the model qualitatively reproduces the experimental results for the OS, BN, and SNR [dashed lines in Fig. 2(c)].

In order to see what happens for lower oscillation amplitudes, we explore the response of hairpin $\mathrm{H} 1$ to an oscillating force of a lower amplitude, $A=0.2 \mathrm{pN}$. A very soft peak and a gentle maximum in the OS and the SNR can be seen around $0.4 \mathrm{~Hz}$ (Fig. S1 in Ref. [18]) in agreement with the results previously obtained for the higher amplitude, $A=0.7 \mathrm{pN}$ (Fig. 2). However, the peak for $A=0.2 \mathrm{pN}$ is much less clear than the peak for $A=0.7 \mathrm{pN}$, showing the importance of using oscillation amplitudes beyond the linear-response regime. $\left(A X^{\dagger \dagger} / k_{B} T \ll 1\right.$, where $X^{\dagger \dagger}$ is the characteristic distance separating the folded or unfolded states from the transition state and $k_{\mathrm{B}}$ is the Boltzmann constant. See also Sec. III in Ref. [18] for SR behavior in the linear-response regime.)

\section{INFLUENCE OF TRAP STIFFNESS AND LENGTH OF THE HANDLES}

An important issue in single-molecule experiments concerns the influence of transducing effects induced by the experimental setup (e.g., trap stiffness and length of the handles) on the measured kinetics. Recent studies $[14,16,17]$ show that the kinetic rates are only moderately affected (within 1 order of magnitude) when the length of the handles is changed 1000-fold or the trap stiffness tenfold. Besides, numerical simulations carried out in Ref. [17] show that kinetic rates for hairpins measured with handles and trap always remain close and converge to the intrinsic rate (i.e., the rate measured without handles and trap) in the limit of very compliant handles. To inquire 
about the influence of the experimental design on the kinetics of hairpin $\mathrm{H} 1$, we have further investigated SR by varying conditions of the experimental setup such as (1) the stiffness of the optical trap and (2) the length of the handles. We have observed how both effects change the intrinsic noise of the system [Figs. 2(b), 2(c), and 3]. In the first case, when the trap stiffness, $\kappa_{\text {trap }}$, is decreased from $70 \mathrm{pN} / \mu \mathrm{m}$ to $24 \mathrm{pN} / \mu \mathrm{m}$ [Fig. 2(b)], the maximum peak in the SNR is shifted to higher frequencies (from $0.4 \mathrm{~Hz}$ to approximately $0.8 \mathrm{~Hz}$ ) and becomes less clear [Fig. 2(c), red curve]. The effect of the trap stiffness on SR is evaluated by using the numerical simulation (Sec. IV in Ref. [18]), and finding good agreement between experiments and simulations [Figs. 2(b) and 2(c)].

In the second case, if we increase by 20 -fold the length of the handles (528 bp and $874 \mathrm{bp}$ at each flanking side) while keeping the trap stiffness constant, $\kappa_{\text {trap }}=$ $70 \mathrm{pN} / \mu \mathrm{m}$, we find that the resonance frequency shifts
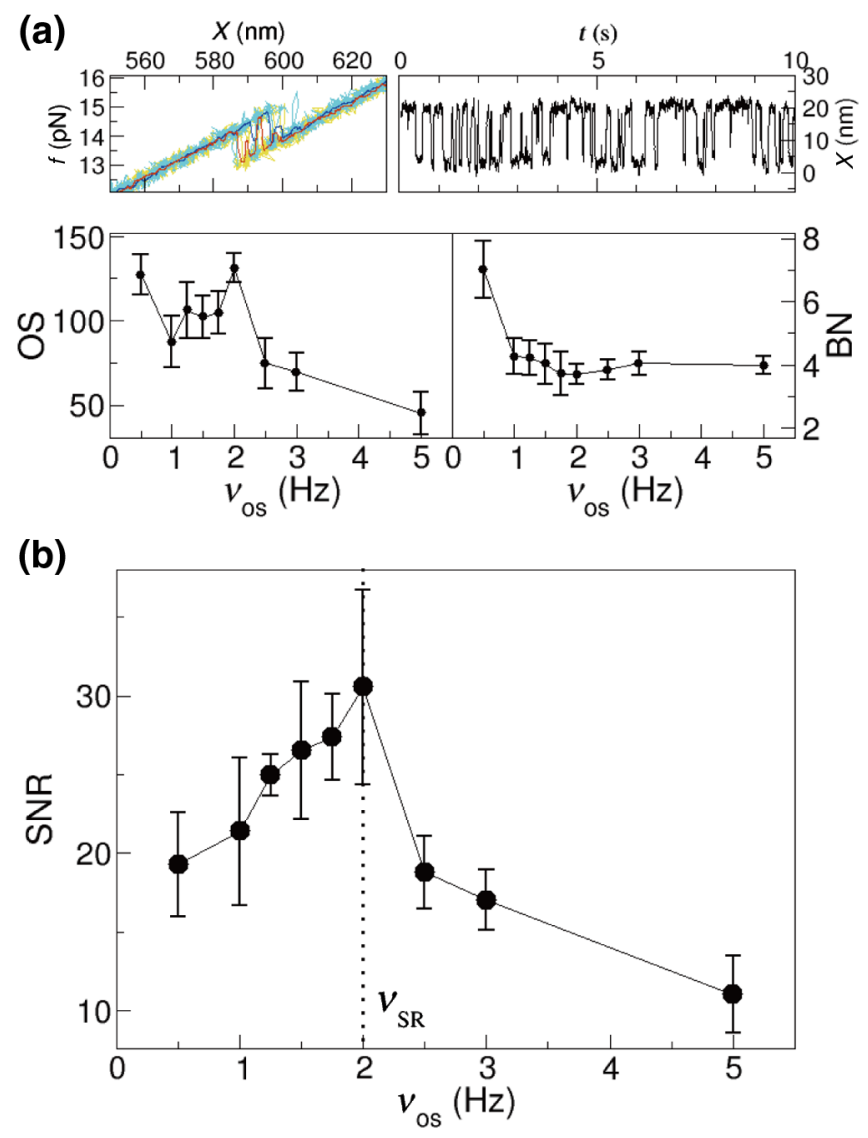

FIG. 3. SR experiments for hairpin $\mathrm{H} 1$ with long DNA handles. (a) Upper left: pulling cycle (unfolding, blue; refolding, red). The dark blue and red curves are the time-averaged data of the paler blue and yellow lines, respectively. Upper right: hopping trace. Lower left: OS. Lower left: BN. (b) The resulting SNR in the case of high trap stiffness $\kappa_{\text {trap }}=70 \mathrm{pN} / \mu \mathrm{m}$ and the amplitude $A=0.7 \mathrm{pN}$. Results have been averaged over 5 molecules. Units: OS $\left(\mathrm{nm}^{2}\right), \mathrm{BN}\left(\mathrm{nm}^{2} / \mathrm{Hz}\right), \mathrm{SNR}(\mathrm{Hz})$. The error bars represent the standard errors over different molecules. to a larger value for the long handles (Fig. 3). For the longhandle construct, the matching condition is again verified $\left(\nu_{\mathrm{SR}}=2 \mathrm{~Hz}\right)$ and $k_{\mathrm{c}} \simeq 4 \mathrm{~s}^{-1}$ as obtained from hopping experiments [14].

The dependence of the resonance frequency measured from SR, $\nu_{\mathrm{SR}}$, on the trap stiffness and the length of the handles is similar to that reported for the hopping rate measured in the hopping experiments at the coexistence force $[14,16,17]$. In both cases, the soft-trap stiffness or the larger compliance of the long handles contributes to increase the hopping rate, supporting the conclusions of Ref. [14]. Interestingly, the quality of the resonant peak worsens as the trap stiffness decreases but not as the linker becomes softer, showing that the quality of the SR peak is dependent only on the combined effective stiffness of bead and handles $\left(\kappa_{\text {eff }}^{-1}=\kappa_{\text {trap }}^{-1}+\kappa_{\text {handle }}^{-1} \simeq \kappa_{\text {trap }}^{-1}\right.$, which is approximately equal to the trap stiffness in our experimental conditions.

\section{OTHER SR QUANTIFIERS}

Next we investigate other representative SR quantifiers. These are the fraction $P_{1}$ of transitions that occur every half-period of the oscillation $[4,24,25]$ and the average dissipated work, $W[5,26]$. To extract $P_{1}$, we measure the residence-time distributions, $P\left(\tau_{\mathrm{F}}\right)$ and $P\left(\tau_{\mathrm{U}}\right)$, of the $\mathrm{F}$ and $\mathrm{U}$ states in the presence of the oscillating force. The distributions are shown in Fig. 4(a) for hairpin $\mathrm{H} 1$ in the cases $\nu_{\mathrm{os}}=0.4 \mathrm{~Hz}$ (upper) and $\nu_{\mathrm{os}}=5 \mathrm{~Hz}$ (lower) with $A=$ $0.7 \mathrm{pN}$. Unlike the distributions shown in Fig. 1(c), $P\left(\tau_{\mathrm{F}}\right)$ $\left[P\left(\tau_{\mathrm{U}}\right)\right]$ is not monotonically decreasing with $\tau_{\mathrm{F}}\left(\tau_{\mathrm{U}}\right)$ and exhibits spikes corresponding to higher harmonics for $\tau_{\mathrm{F}}=T_{\mathrm{os}}(1+2 n) / 2 \quad\left(\tau_{\mathrm{U}}=T_{\mathrm{os}}(1+2 n) / 2\right) \quad$ where $n=$ $0,1,2, \cdots$. A few harmonic frequencies are shown as vertical arrows in Fig. 4(a). In particular, when $\nu_{\mathrm{os}}$ is close to the resonance frequency, the shape of the residence-time distribution strongly deviates from an exponential, and a broad peak appears around the fundamental mode, $\tau_{\mathrm{F}}=$ $T_{\mathrm{os}} / 2\left(\tau_{\mathrm{U}}=T_{\mathrm{os}} / 2\right)$ [Fig. 4(a), top]. In contrast, many peaks appear in the residence-time distribution when $\nu_{\text {os }} \gg \nu_{\text {SR }}$ [Fig. 4(a), lower].

$P_{1}$ can be extracted from the area of the residence-time distribution around the peak located at the fundamental mode, $\tau_{\mathrm{F}}=T_{\mathrm{os}} / 2\left(\tau_{\mathrm{U}}=T_{\mathrm{os}} / 2\right)$. Let $\left\{\tau_{i} ; i=1, \cdots, N\right\}$ be the series of $N$ residence times measured in the presence of the oscillating force. By counting the number, $n$, of $\tau_{i}$ that satisfy the condition $T_{\mathrm{os}} / 2-T_{\mathrm{os}} / 4 \leq \tau_{i} \leq T_{\mathrm{os}} / 2+$ $T_{\text {os }} / 4$, we define

$$
P_{1}=\frac{n}{N}
$$

$P_{1}$ takes a large value if the residence time of the hairpin is equal to half the period of the oscillating force. This means that a large fraction of hopping transitions occur when the oscillating force changes sign. Therefore, the value of $P_{1}$ has a maximum when SR is induced, because the transitions between the two states are synchronized with the 


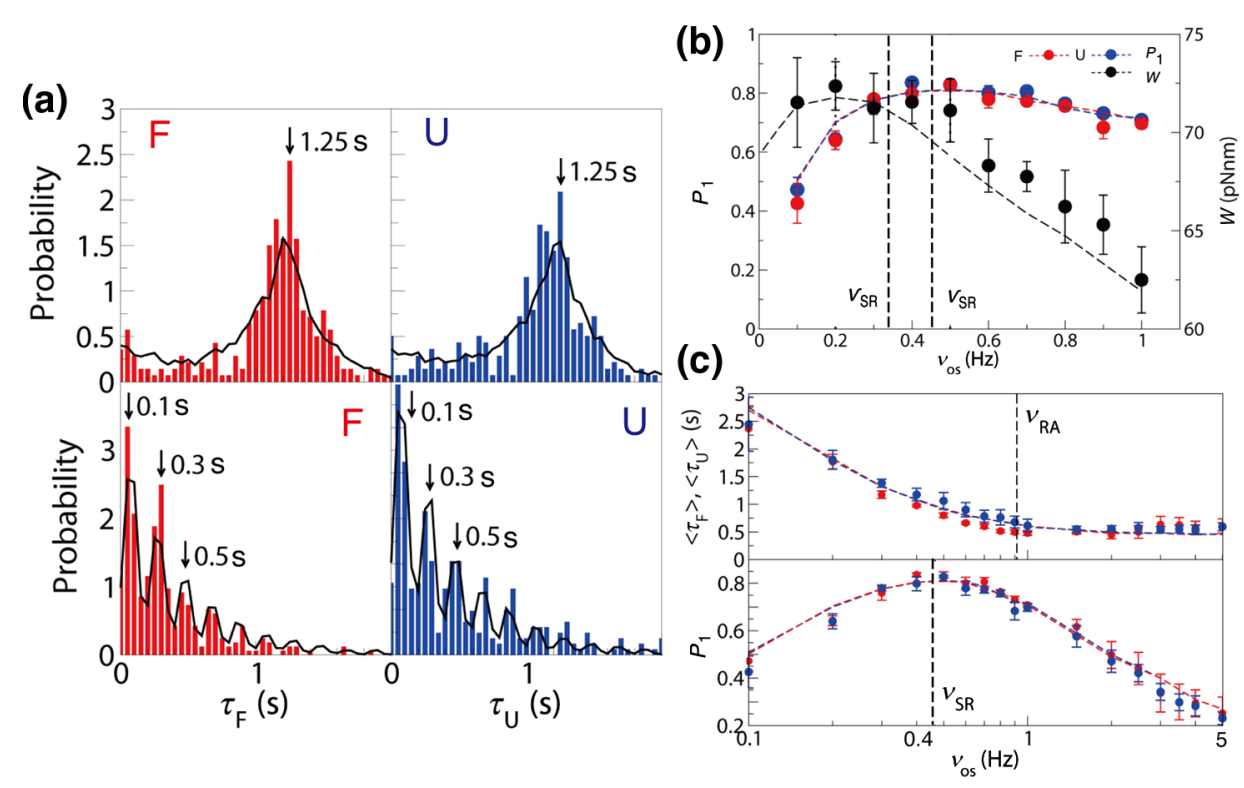

FIG. 4. Other SR quantifiers in hairpin H1. (a) Residence-time distributions for the F (red) and U (blue) states at $\nu_{\mathrm{os}}=0.4 \mathrm{~Hz}$ (upper) and $\nu_{\mathrm{os}}=5 \mathrm{~Hz}$ (lower). The continuous black lines are the results of the simulations (Sec. IV in Ref. [18]). (b) $P_{1}$ in the F (red) and $U$ (blue) and average work $W$ (black) as a function of input frequency. Simulation results are shown by the dashed lines (Sec. IV in Ref. [18]). Vertical dashed lines show the expected resonance frequency. The maximum in $P_{1}$ is broad around $0.3-0.6 \mathrm{~Hz}$, while that in $W$ is also broad with a maximum found at a lower frequency in the range $0.1-0.4 \mathrm{~Hz}$. (c) Average residence times (upper panel) and $P_{1}$ (lower panel) in the F (red) and $\mathrm{U}$ (blue) states as a function of input frequency. Note that the frequency range is larger than that shown in (b). Simulation results are shown as dashed curves (Sec. IV in Ref. [18]). The vertical dashed lines show the two frequencies characteristic of stochastic resonance $\left(\nu_{\mathrm{SR}}\right)$ and resonant activation $\left(\nu_{\mathrm{RA}}\right)$. Statistics: 8 molecules, 3-5-minute traces, and 300-600 hopping transitions at each input frequency. The error bars represent the standard errors over different molecules.

oscillating force. $\left(P_{1}\right.$ is a bona-fide SR quantifier [24]. See also Sec. III in Ref. [18].) The results obtained for $P_{1}$ in hairpin $\mathrm{H} 1$ are shown in Fig. 4(b). $P_{1}$ exhibits a broad maximum around the resonance value $\nu_{\mathrm{SR}}=k_{\mathrm{c}} / 2=$ $0.4 \mathrm{~Hz}$. The broadness of the peak is in contrast to the narrower peak observed in the SNR [Fig. 2(c)]. These results are consistent with analytical calculations [12,24].

For the average cyclic work done by an oscillating force, we define [27]

$$
W=-\langle\oint X d f\rangle=\langle\oint f d X\rangle,
$$

where the brackets stand for statistical averages over traces. Because $W$ takes a large value when the folding or unfolding of the hairpin is synchronized with the oscillating force, it is a useful SR quantifier as well $[5,28]$. In fact, the stronger the synchronization between transitions of the hairpin and oscillations in the force, the larger the work done by the optical trap on the molecule. Results for $W$ are shown in Fig. 4(b). In contrast to SNR but similar to $P_{1}$, the maximum in $W$ is broad. Finally, we compare our experimental results with the predictions obtained from the numerical simulations in the continuous double-well potential whose parameters are the same as those used in Fig. 2 (Sec. IV in Ref. [18]). Figures 4(a) and 4(b) show a good agreement between experiments and simulations. Although both $P_{1}$ and $W$ show broad maxima as a function of $\nu_{\mathrm{os}}$, the positions of their maxima do not coincide with each other; the maximum for the work is found at a lower frequency as compared to $P_{1}$. As we pointed out in the introduction, the precise value of the resonance frequency depends on the choice of the quantifier used, especially when the quality of the resonant peak is low.

\section{RESONANT ACTIVATION}

In stochastic systems driven by oscillating forces, it is customary to distinguish two effects: stochastic resonance (SR) and resonant activation (RA). SR is the optimization of the response of the system (i.e., the output signal) whereas RA is the optimization of kinetics (e.g., maximization of the number of hopping transitions per second). SR and RA are different phenomena related to barriercrossing dynamics along temporally modulated energy landscapes [4]. RA is induced when the mean residence times of the states of the system are minimized with respect to the frequency of the oscillating force, at $\nu_{\mathrm{RA}}$. The values of $\nu_{\mathrm{SR}}$ and $\nu_{\mathrm{RA}}$ are often not the same, the latter being typically larger than the former. Figure 4(c) (top) shows the mean residence times, $\left\langle\tau_{\mathrm{F}}\right\rangle$ and $\left\langle\tau_{\mathrm{U}}\right\rangle$, for hairpin $\mathrm{H} 1$ measured in the range $0.1 \mathrm{~Hz} \leq \nu_{\mathrm{os}} \leq 5 \mathrm{~Hz}$. Only at higher frequencies (between $1 \mathrm{~Hz}$ and $2 \mathrm{~Hz}$ ), the graph suggests a very shallow minimum for the residence times. Therefore, we are capable of observing both the SR and RA phenomena in the single-molecule experiments. The experimental results also agree with the numerical simulations 
[Fig. 4(c), dashed vertical lines]. Similar behavior has been reported in the experiments with a colloidal particle in a double-well potential generated by optical tweezers [4].

\section{SR IN SHORTER HAIRPINS}

SR might be used to detect the transitions between $\mathrm{F}$ and $\mathrm{U}$ states in cases where the hopping events of a hairpin are hard to discriminate. In these cases, the hopping signals (extension jumps) are on the same order as the standard deviation of noise fluctuations. To investigate this problem, we design two short hairpins (SH10 and SH8) having only 10 and 8 base pairs along the stem, respectively [sequences shown in Figs. 5(c) and 5(d)]. The molecular free-energy landscapes are calculated for the two sequences at the theoretically predicted coexistence forces using the nearest-neighbor model for DNA [Fig. 5(a), upper left] $[29,30]$. As the length of the stem decreases, the landscapes show progressively lower coexistence force values, molecular extensions, and kinetic barriers. Measurements for SH10 and SH8 are taken at low trap stiffnesses to decrease the hopping signal $\left(\kappa_{\text {trap }} \simeq 32 \mathrm{pN} / \mu \mathrm{m}\right.$ and $17 \mathrm{pN} / \mu \mathrm{m}$, respectively). Pulling curves and hopping traces in the CFM are also shown in Fig. 5(a) (lower left). While the transitions are still visible for SH10, they are hardly distinguishable for SH8. Similar observations are also apparent from the dwell distributions on trap position, $X$, shown in Fig. 5(a) (right). Measured jumps in the molecular extension upon unfolding (a)
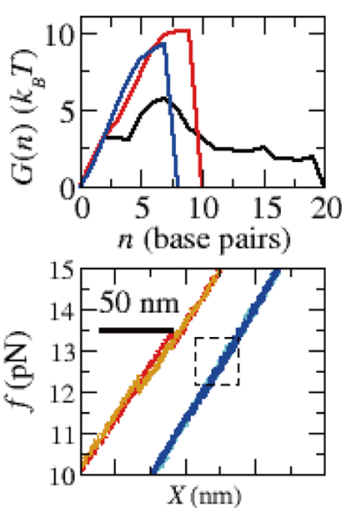

(c)
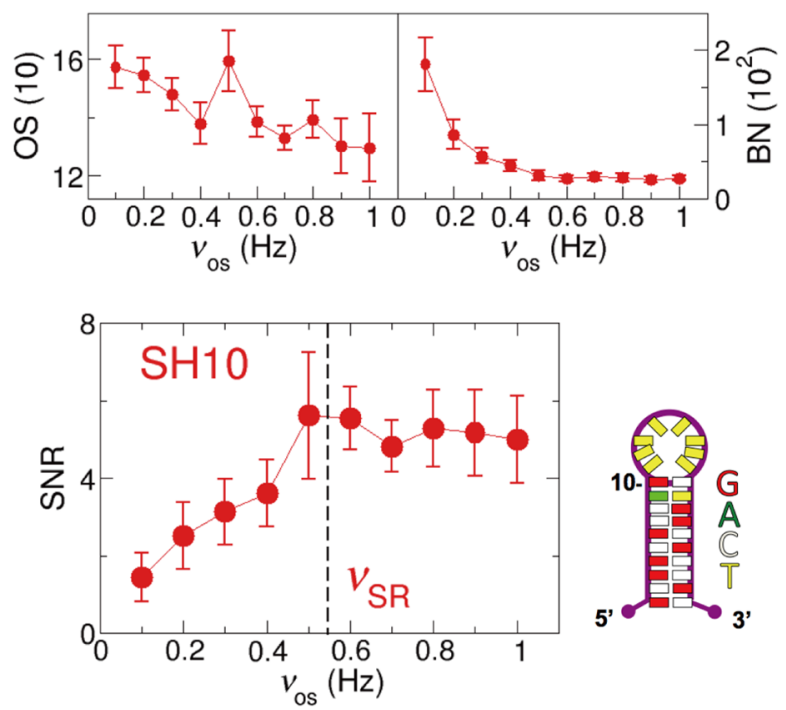

(b)
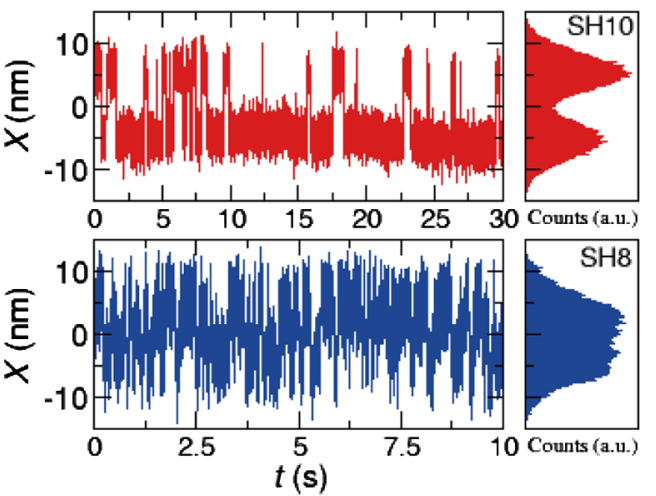

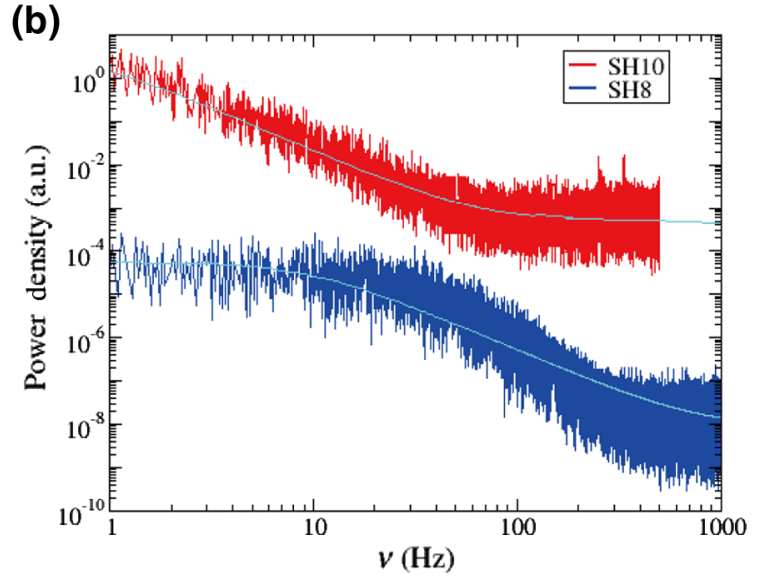

(d)
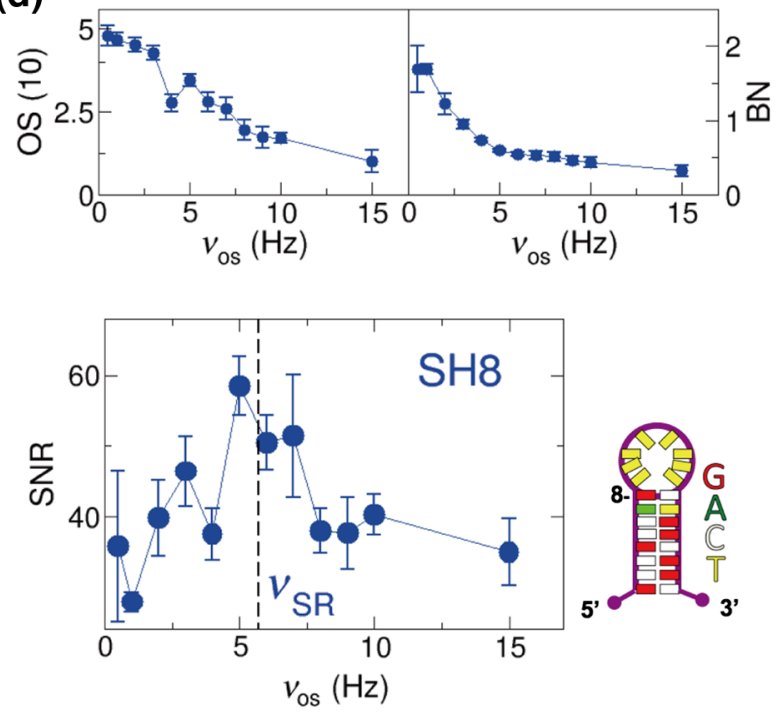

FIG. 5. SR experiments in shorter hairpins. (a) Free-energy landscapes (upper left), force-distance curves (lower left), and hopping traces in the CFM for SH10 (red), SH8 (blue), and H1 (black). Measurements have been carried out with a low trap power $\kappa_{\text {trap }} \simeq$ $32 \mathrm{pN} / \mu \mathrm{m}$ for SH10 and $17 \mathrm{pN} / \mu \mathrm{m}$ for SH8, respectively. For simplicity, all pulling curves in the lower left panel are shown parallel with equal average slopes. The dashed square region for SH8 curves indicates the region where unfolding and folding transitions occur. Distributions of trap position, $X$, show clear transitions for SH10 but not for SH8. (b) Power spectrum for SH10 and SH8. Cyan curves are fits to a sum of two Lorentzians (see text for details). Colors mean the same as in (a). (c),(d) OS, BN, and SNR for SH10 and SH8. The amplitudes of oscillation force are $A=0.5 \mathrm{pN}$ for SH10 and $A=0.15 \mathrm{pN}$ for SH8, respectively. Colors mean the same as in (a). Statistics (SH10, SH8): molecules (5, 7); duration of hopping traces (4, 2 minutes); average number of hopping transitions $(250,1200)$ at each input frequency. Units: OS $\left(\mathrm{nm}^{2}\right), \mathrm{BN}\left(\mathrm{nm}^{2} / \mathrm{Hz}\right)$, SNR $(\mathrm{Hz})$. The error bars represent the standard errors over different molecules. 
or folding are equal to $10.5 \pm 0.5 \mathrm{~nm}$ and $7.0 \pm 0.5 \mathrm{~nm}$ for SH10 and SH8, respectively.

Figure 5(b) shows the power spectra of $X(t)$. Whereas SH10 can be fit reasonably well to a sum of two Lorentzians with two characteristic corner frequencies $(0.64 \pm 0.02 \mathrm{~Hz}$ and $2.4 \pm 0.3 \mathrm{kHz})$, the quality of the fit considerably worsens for SH8 (approximately $9.8 \mathrm{~Hz}$ and $15.6 \mathrm{kHz}$ ). The low frequency (in the range of $\mathrm{Hz}$ ) in the power spectra of SH8 corresponds to the hopping kinetics of the hairpin, whereas the higher frequency (in the range of $\mathrm{kHz}$ ) corresponds to the random motion of the optical trap caused by the force feedback. Because the noise in the trap position, $X$, introduced by the force-feedback protocol is not of thermal origin, the power spectra measured in the CFM should not necessarily be fitted to a sum of two Lorentzians. This problem with the two-Lorentzian fit is especially acute for SH8 where the feedback loop cannot follow the fast hopping transitions.

Once the hopping properties of the hairpins are characterized, we then carry out the oscillating experiments for hairpins SH10 and SH8 around the coexistence force. The results we obtain for SH10 are similar to those reported for hairpin $\mathrm{H} 1$ at low trap power shown in Fig. 2(c). For SH10, the peak in the SNR around $\nu_{\mathrm{SR}}=0.5 \mathrm{~Hz}$ is close to $k_{\mathrm{c}} / 2$ where $k_{\mathrm{c}}$ is measured to be $0.43 \pm 0.07 \mathrm{~s}^{-1}$ from the hopping traces for $X(t)$. More interesting is the case of hairpin SH8 where the coexistence force can still be located, but the hopping signal is blurred by the fluctuations. In Fig. 5(d), we can see that the OS and the SNR exhibit a maximum around $\nu_{\mathrm{SR}}=5 \pm 1 \mathrm{~Hz}$ for SH8, which gives $k_{\mathrm{c}} \simeq 10 \pm 2 \mathrm{~s}^{-1}$ according to the matching condition. This value agrees with the value of $9.8 \mathrm{~Hz}$ obtained from the Lorentzian fit to the power spectrum. As an additional test, we have implemented a hidden Markov model (HMM) with the forward-backward feedback algorithm as described in Ref. [31] to extract the kinetic rates of SH8 from the hopping trace, $X(t)$. By applying the HMM to the hopping traces of SH8, we obtain a value of $k_{\mathrm{c}}=9.4 \pm 0.5 \mathrm{~s}^{-1}$ (averaged over seven molecules), which confirms the results obtained with SR and Lorentzian fit to the spectral density.

Thus, SR offers an alternative method of estimating the hopping rate of SH8. Indeed, the two states (F and U) cannot be easily detected from the hopping trace, and the residencetime analysis done for hairpin H1 [Fig. 1(c)] is difficult to implement. In this case, SR confirms the value of the hopping frequency initially obtained from a poor Lorentzian fit of the power spectrum. Moreover, although the power spectra shown in Fig. 5(b) can be fitted by a sum of two Lorentzians, it becomes impossible to distinguish these two in the case of shorter hairpins. There is a possibility that SR can be used to find the hopping rate even in this case.

\section{CONCLUSION}

We have carried out SR experiments in single DNA hairpins subject to an oscillatory mechanical force of varying frequency. Our aim is to investigate how a molecule exhibiting bistability (i.e., hopping between the folded and unfolded conformations) responds to an applied oscillating force. In SR, the response gets amplified at frequencies close to the characteristic hopping frequency of the hairpin. By measuring the power spectral density of the molecular extension, we have carried out a detailed investigation of the frequency dependence of the output signal [OS, Eq. (2)], the background noise [BN, Eq. (3)], and the signal-to-noise ratio [SNR, Eq. (4)] in the 20-bp hairpin H1 which exhibits dichotomous hopping behavior. We then have extended our research by exploring how several parameters of the experimental setup such as trap stiffness, length of the handles, oscillating amplitude, and size of the hairpin influence the resonance behavior. From the measured traces, we have also analyzed a few other SR quantifiers, such as the number of folding and unfolding transitions occurring every halfperiod of the oscillation $\left[P_{1}\right.$, Eq. (5)], the average mechanical work per period of the oscillation [W, Eq. (6)], and the mean residence times in the unfolded and folded states $\left(\left\langle\tau_{\mathrm{U}}\right\rangle\right.$ and $\left.\left\langle\tau_{\mathrm{F}}\right\rangle\right)$. The mean residence times describe a mechanism slightly different from SR that has been termed resonant activation (RA). Overall, we find that the SNR and the other SR quantifiers (such as OS, $P_{1}$, $W$ ) exhibit a peak at a frequency close to that determined by the resonance-matching condition. Among all quantifiers, only the SNR and the OS tend to show a modest amplification of the response, with the SNR showing a higher-quality peak. Our results are summarized in Table I. Moreover, our experimental results are well predicted by numerical simulations of an overdamped particle in a double-well potential reproducing the measured molecular free-energy landscape of the hairpin (Sec. IV in Ref. [18]). Finally, our experimental findings also agree with theoretical results [11] that show a modest gain in the response of noisy systems driven by oscillating forces.

A unique aspect of our work is the investigation of SR in small systems in conditions of weak thermodynamic stability (folding free energies of a few $k_{B} T$ units) not far from noise level $\left(k_{B} T\right)$. This has a primary consequence: The proper control parameter in our experiments does not appear to be the noise intensity. In fact, by changing noise intensity (e.g., by tuning temperature or denaturant concentration), we also modify the structural properties of the molecule in a noncontrolled way (i.e., by changing its thermodynamic stability or free energy of formation). Our work circumvents this problem by using the frequency of the external driving force as control parameter. Simple as this choice may seem, only a few theoretical and experimental works have addressed it in the past $[11,20]$. From this perspective, our study should stimulate further theoretical work in the SR of small systems where noise intensity and thermodynamic stability are tightly coupled. Another consequence of the noise intensity vs thermodynamic stability 
TABLE I. Comparison between $\nu_{\mathrm{SR}}(\mathrm{Hz})$ and $k_{\mathrm{c}}\left(\mathrm{s}^{-1}\right)$. Resonance frequency, $\nu_{\mathrm{SR}}$, obtained from SNR, OS, $P_{1}$, and $W$ vs hopping rate, $k_{\mathrm{c}}$, at the coexistence force (see Sec. I in Ref. [18] for $k_{\mathrm{c}}$ ). $\nu_{\mathrm{SR}}$ was chosen as the peak value of each SR quantifier for each molecule. $n=$ the number of molecules analyzed.

\begin{tabular}{|c|c|c|c|c|c|}
\hline & $\nu_{\mathrm{SR}}$ from SNR & $\nu_{\mathrm{SR}}$ from OS & $\nu_{\mathrm{SR}}$ from $P_{1}$ & $\nu_{\mathrm{SR}}$ from $W$ & $k_{\mathrm{c}} / 2$ \\
\hline $\mathrm{H} 1^{\mathrm{a}, \mathrm{c}}$ & $0.40 \pm 0.02(n=10)$ & $0.45 \pm 0.03(n=10)$ & $\begin{array}{c}0.45 \pm 0.04^{\mathrm{e}} \\
0.48 \pm 0.08^{\mathrm{f}}(n=8)\end{array}$ & $0.33 \pm 0.07(n=8)$ & $0.33 \pm 0.02(n=12)$ \\
\hline $\mathrm{H} 1{ }^{\mathrm{a}, \mathrm{d}}$ & $0.72 \pm 0.08(n=5)$ & $0.50 \pm 0.06(n=5)$ & & & $0.53 \pm 0.07(n=8)$ \\
\hline $\mathrm{H} 1^{\mathrm{b}, \mathrm{c}}$ & $2.0 \pm 0.2(n=5)$ & $1.9 \pm 0.1(n=5)$ & & & $2.2 \pm 0.3(n=5)$ \\
\hline SH10 & $0.54 \pm 0.02(n=5)$ & $0.58 \pm 0.04(n=5)$ & & & $0.43 \pm 0.07(n=4)$ \\
\hline SH8 & $5.7 \pm 0.4(n=7)$ & $5.3 \pm 0.2(n=7)$ & & & $4.7 \pm 0.3^{\mathrm{g}}(n=7)$ \\
\hline
\end{tabular}

${ }^{\mathrm{a}}$ Short handles

${ }^{\mathrm{b}}$ Long handles

${ }^{\mathrm{c}}$ High power trap $(\kappa=70 \mathrm{pN} / \mu \mathrm{m})$

${ }^{\mathrm{d}}$ Low power trap $(\kappa=24 \mathrm{pN} / \mu \mathrm{m})$

${ }^{\mathrm{e}}$ Folded state

${ }^{\mathrm{f}}$ Unfolded state

${ }^{\mathrm{g}}$ Rate determined using a hidden Markov model

coupling is the strong variability exhibited by singlemolecule SR experiments: The measured SNR versus any control parameter (in our case, oscillation frequency) tends to show large variations from molecule to molecule. This is apparent in the results for hairpin H1 and SH8 shown in Fig. 6 and has been observed in the rest of molecules. Such variability is a consequence of the aforementioned weak stability of biomolecular bonds and of various sources of experimental errors (e.g., instrumental drift, misalignment attachment, inaccurate discrimination of the coexistence force, etc.). This variability has no counterpart in other SR studies of nonlinear macroscopic devices or single-degree-
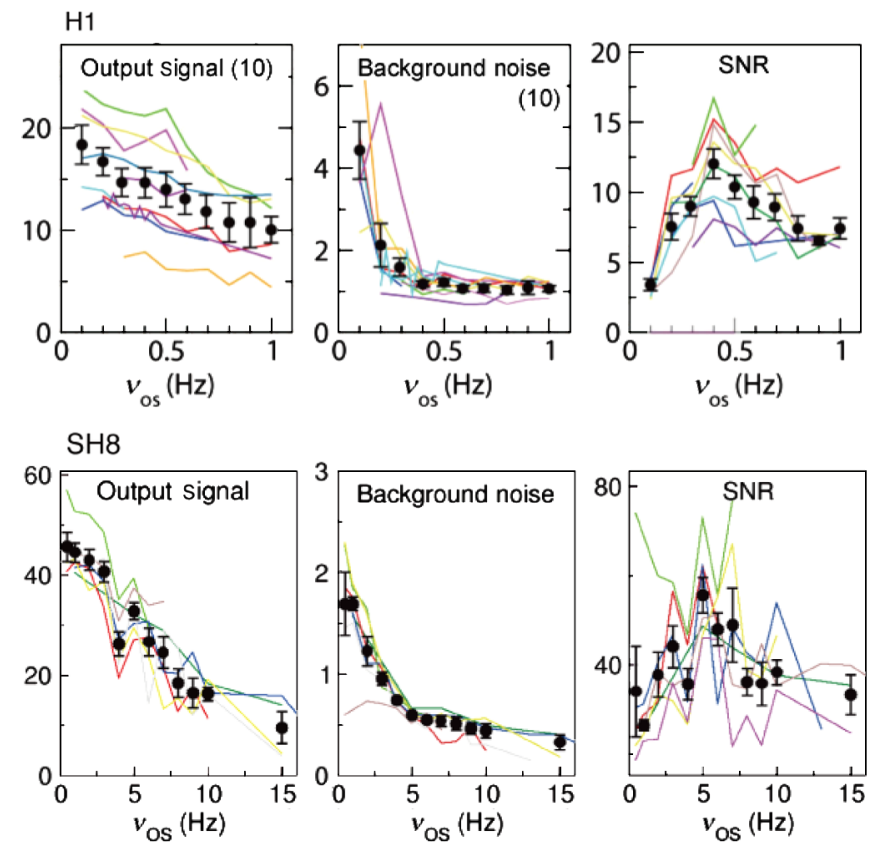

FIG. 6. Molecular variability of the measured responses (H1, SH8). Results are for the OS, BN, and SNR for different molecules. Units: OS $\left(\mathrm{nm}^{2}\right), \mathrm{BN}\left(\mathrm{nm}^{2} / \mathrm{Hz}\right), \mathrm{SNR}(\mathrm{Hz})$. The error bars represent the standard error over different molecules. of-freedom systems (such as a single colloidal particle in optical traps or macroscopic systems in solid-state physics or electronic devices).

\section{FUTURE PERSPECTIVES}

The results of our work suggest that we could extract the kinetic rates of molecular hoppers by measuring the resonance frequency in experiments involving oscillating driving forces. Is this approach useful? There are several widely accepted and commonly used single-molecule methods that can extract the kinetic parameters of molecular hoppers just by analyzing the hopping traces without the need to carry out oscillating measurements. It is then clear that single-molecule SR is not worth pursuing if other simpler methods are available. Yet SR might be of interest for investigating fast molecular transitions where current methods might fail. In Sec. VII, we have investigated SR in an 8-bp short DNA hairpin (SH8) at conditions (low trap stiffness) where hopping rates are hard to measure by standard methods (e.g., the Bell-Evans model). The faster hopping rate and the smaller jumps in extension (due to both the shorter length of SH8 and the decreased trap stiffness) contribute to making the hopping-rate measurements difficult. Note that we have been able to extract the value of the hopping rate either by measuring the power spectrum [Fig. 5(b)] or by implementing a hidden Markov model. Interestingly, whereas applying standard methods to extract kinetic rates becomes steadily difficult as the hopping signal becomes more noisy, the quality of the resonant peak in the SNR remains acceptable [Fig. 5(d)]. This suggests that, in experimental conditions where hopping signals become nearly undetectable, SR may find fertile ground for useful applications.

Measuring the kinetics of single bonds might be crucial to dissecting the kinetic pathways of many reactions, from nucleic-acid translocases indispensable in virtually all tasks of nucleic-acids metabolism, to molecular folding 
of proteins and ligand-receptor binding. Moreover, the detection of single-bond kinetics also provides a direct measurement of the affinity (or free energy of formation) of weak single bonds (important, e.g., for an accurate determination of the parameters characterizing the thermodynamics of secondary structure formation in nucleic acids [32]). It is therefore important to explore new approaches capable of illuminating such questions. The experimental resolution of formation and dissociation kinetics is currently limited to five base pairs $[29,33]$. Overcoming this limit strongly relies not only on increasing the hopping signal relative to the noise but also in slowing down the formation and dissociation kinetics of single bonds that are expected to be too fast. A direct measurement of the formation and dissociation fast kinetics of single molecular bonds stretchable on subnanometer scales and resistant to low (a few piconewton) forces remains an experimental challenge.

In fact, the route to discriminating hopping kinetics in a small number of base pairs may be plagued by difficulties. The situation might be even worse if the aim is to detect the unraveling kinetics of a single nearest-neighbor base pair (NNBP), which is the minimal unit of DNA bonds. (Double-stranded helices are stabilized both by hydrogen bonds between complementary bases and by stacking between NNBPs.) Currently, most kinetics measurements are carried out in hopping experiments. However, there is a complication in hopping experiments due to the low signal-to-noise ratio inherent in unraveling a single NNBP together with the disturbances caused by the multifrequency noise present in the high-frequency range where the kinetic rate of formation and dissociation of a single NNBP is expected to fall. The low signal-to-noise-ratio problem can be partially resolved using advanced dataanalysis tools such as Bayesian methods and HMM, as was done in our analysis of the hopping traces for SH8. However, such methods assume a specific form of the noise (i.e., decorrelated force fluctuations and Gaussian emission signal) and do not account for multifrequency sources of noise (due to the aforementioned sources). In this regard, SR might be useful for separating the true formation and dissociation kinetics of a single NNBP from these other artifacts.

Finally, while our work focused on the SR phenomenon in DNA hairpins, other interesting molecular structures are now available for single-molecule mechanical experiments. It would be very interesting to carry out SR measurements in more complex molecular folders (e.g., those exhibiting multiple folding pathways, intermediate states, or noncooperative transitions) such as RNAs and proteins.

\section{ACKNOWLEDGMENTS}

K.H. is supported by a Grant-in-Aid for Scientific Research from the MEXT (No. 23107703). M. M. is supported by Juan de la Cierva Program MICINN-JDC and EU (BioNanoSwitch). J. M. H. and F. R. are supported by
Grant No. FIS2007-3454, ICREA Academia 2008, and HFSP No. RGP0055-2008.

\section{APPENDIX A: METHODS}

\section{Synthesis of DNA hairpins}

The DNA hairpins with handles are synthesized using the hybridization of three different oligonucleotides [Fig. 1(a)]. One oligonucleotide contains the sequence of the ssDNA left handle plus a part of the sequence of the desired DNA hairpin; the second has the rest of the sequence of the DNA hairpin and the ssDNA right handle. The right and left handles have the same sequence in order to hybridize them with the third oligonucleotide. The first oligonucleotide has a biotin at its 5' end and the second oligonucleotide has been modified at its 3 ' end with a digoxigenin tail (DIG Oligonucleotide Tailing Kit, 2nd generation, Roche Applied Science, Indianapolis, Indiana, USA). Once the first and second oligonucleotides are hybridized to form the hairpin, the third oligonucleotide is hybridized to the handles to form the dsDNA handles. Streptavidin-coated polystyrene microspheres $(1.87 \mu \mathrm{m}$; Spherotech, Libertyville, Illinois, USA) and protein G microspheres (3.0-3.4 $\mu \mathrm{m}$; G. Kisker Gbr, Products for Biotechnology, Steinfurt, Germany) coated with antidigoxigenin polyclonal antibodies (Roche Applied Science) are used for specific attachments to the DNA molecular constructions described above. Attachment to the antidigoxigenin microspheres is achieved first by incubating the beads with the tether DNA. The second attachment is accomplished by bringing a trapped antidigoxigenin and streptavidin microspheres close to each other. The sequences of the short hairpins are SH10 (5'-GCGGCGCCAG TTTTTTTTCTGGCGCCGC-3') and SH8 (5'-GGCGCC AGTTTTTTTTCTGGCGCC-3').

\section{Experimental setup}

The experiments have been carried out using a highstability, newly designed apparatus of miniaturized dualbeam optical tweezers [32]. The apparatus consists of two counterpropagating laser beams of $845-\mathrm{nm}$ wavelength that form a single optical trap in which particles can be trapped by gradient forces. The DNA hairpin is tethered between two beads [Fig. 1(a)]. One bead is immobilized at the tip of a micropipette that is glued to the fluidics chamber; the optical trap captures the other bead. The light deflected by the bead is collected by two photodetectors located at opposite sides of the chamber. They directly measure the total change in light momentum which is equal to the net force acting on the bead. Piezo actuators bend the optical fibers and allow the user to move the optical trap. The force is made to oscillate using a force-feedback system that operates at $4 \mathrm{kHz}$, minimizing instrumental-drift effects as compared to protocols without feedback. Force feedback does not introduce artifacts in 
our measurements unless $\nu_{\mathrm{os}}$ is too high (typically larger than $50 \mathrm{~Hz}$ ) or $A$ is too small (less than $0.1 \mathrm{pN}$ ).

The folding and unfolding experiments described in this paper were performed at room temperature $\left(24^{\circ} \mathrm{C}\right)$ in a buffer containing $10 \mathrm{mM}$ Tris- $\mathrm{HCl}$ at $\mathrm{pH} 7.5,1 \mathrm{mM}$ ethylenediaminetetraacetic acid (EDTA), $1 \mathrm{M} \mathrm{NaCl}$, and $0.01 \%$ sodium azide.

[1] R. Benzi, A. Sutera, and A. Vulpiani, The Mechanism of Stochastic Resonance, J. Phys. A 14, L453 (1981).

[2] R. Benzi, G. Parisi, A. Sutera, and A. Vulpiani, Stochastic Resonance in Climatic Changes, Tellus 34, 10 (1982).

[3] A. Simon and A. Libchaber, Escape and Synchronization of a Brownian Particle, Phys. Rev. Lett. 68, 3375 (1992).

[4] C. Schmitt, B. Dybiec, P. Hänggi, and C. Bechinger, Stochastic Resonance vs. Resonant Activation, Europhys. Lett. 74, 937 (2006).

[5] P. Jop, A. Petrosyan, and S. Ciliberto, Work and Dissipation Fluctuations near the Stochastic Resonance of a Colloidal Particle, Europhys. Lett. 81, 50005 (2008).

[6] D.F. Russell, L. A. Wilkens, and F. Moss, Use of Behavioural Stochastic Resonance by Paddle Fish for Feeding, Nature (London) 402, 291 (1999).

[7] M. McDonnell and D. Abbott, What Is Stochastic Resonance? Definitions, Misconceptions, Debates, and Its Relevance to Biology, PLoS Comput. Biol. 5, e1000348 (2009).

[8] D. Petracchi, M. Pellegrini, M. Pellegrino, M. Barbi, and F. Moss, Periodic Forcing of a $\mathrm{K}^{+}$Channel at Various Temperatures, Biophys. J. 66, 1844 (1994).

[9] M. Grifoni and P. Hänggi, Coherent and Incoherent Quantum Stochastic Resonance, Phys. Rev. Lett. 76, 1611 (1996).

[10] D. Witthaut, F. Trimborn, and S. Wimberger, DissipationInduced Coherence and Stochastic Resonance of an Open Two-Mode Bose-Einstein Condensate, Phys. Rev. A 79, 033621 (2009).

[11] N.G. Stocks, A Theoretical Study of the Non-Linear Response of a Periodically Driven Bistable System, Nuovo Cimento D 17, 925 (1995).

[12] L. Gammaitoni, P. Hänggi, P. Jung, and F. Marchesoni, Stochastic Resonance, Rev. Mod. Phys. 70, 223 (1998).

[13] T. Wellens, V. Shatokhin, and A. Buchleitner, Stochastic Resonance, Rep. Prog. Phys. 67, 45 (2004).

[14] N. Forns, S. de Lorenzo, M. Manosas, K. Hayashi, J. M. Huguet, and F. Ritort, Improving Signal-to-Noise Resolution in Single Molecule Experiments Using Molecular Constructs with Short Handles, Biophys. J. 100, 1765 (2011).

[15] For 29-bp molecular handles (approximately $10 \mathrm{~nm}$ of contour length at each flanking side), the molecular extension is short (approximately $20 \mathrm{~nm}$ ), and the beads are very close to each other. However, the strong stiffness of the handle under tension prevents the beads from clashing [14].

[16] J.-D. Wen, M. Manosas, P.T.X. Li, S. B. Smith, C. Bustamante, F. Ritort, and I. Tinoco, Force Unfolding Kinetics of RNA Using Optical Tweezers. I. Effects of
Experimental Variables on Measured Results, Biophys. J. 92, 2996 (2007).

[17] M. Manosas, J.-D. Wen, P.T.X. Li, S. B. Smith, C. Bustamante, I. Tinoco, and F. Ritort, Force Unfolding Kinetics of RNA Using Optical Tweezers. II. Modeling Experiments, Biophys. J. 92, 3010 (2007).

[18] See Supplemental Material at http://link.aps.org/ supplemental/10.1103/PhysRevX.2.031012 for kinetic rates of hairpins, experimental results in the case $A=$ $0.2 \mathrm{pN}, \mathrm{SR}$ in a discrete two-state system, and numerical simulations.

[19] B. McNamara and K. Wiesenfeld, Theory of Stochastic Resonance, Phys. Rev. A 39, 4854 (1989).

[20] A. L. Pankratov, Suppression of Noise in Nonlinear Systems Subjected to Strong Periodic Driving, Phys. Rev. E 65, 022101 (2002).

[21] V. Berdichevsky and M. Gitterman, Stochastic Resonance in a Bistable Piecewise Potential: Analytical Solution, J. Phys. A 29, L447 (1996).

[22] M.C. Mahato and A. M. Jayannavar, Some Stochastic Phenomena in a Driven Double-Well System, Physica A (Amsterdam) 248, 138 (1998).

[23] J. Casado-Pascual, J. Gomez-Ordoñez, M. Morillo, and P. Hänggi, Two-State Theory of Nonlinear Stochastic Resonance, Phys. Rev. Lett. 91, 210601 (2003).

[24] L. Gammaitoni, F. Marchesoni, and S. Santucci, Stochastic Resonance as a Bona Fide Resonance, Phys. Rev. Lett. 74, 1052 (1995).

[25] T. Zhou, F. Moss, and P. Jung, Escape-Time Distributions of a Periodically Modulated Bistable System with Noise, Phys. Rev. A 42, 3161 (1990).

[26] S. Saika, R. Ratnadeep, and A. M. Jayannavar, Work Fluctuations and Stochastic Resonance, Phys. Lett. A 369, 367 (2007).

[27] A. Mossa, S. De Lorenzo, J. M. Huguet, and F. Ritort, Measurement of Work in Single Molecule Experiments, J. Chem. Phys. 130, 234116 (2009).

[28] P. Jung and F. Marchesoni, Energetics of Stochastic Resonance, Chaos 21, 047516 (2011).

[29] M. T. Woodside, W. M. Behnke-Parks, K. Larizadeh, K. Travers, D. Herschlag, and S. M. Block, Nanomechanical Measurements of the Sequence-Dependent Folding Landscapes of Single Nucleic Acid Hairpins, Proc. Natl. Acad. Sci. U.S.A. 103, 6190 (2006).

[30] A. Mossa, M. Manosas, N. Forns, J. M. Huguet, and F. Ritort, Dynamic Force Spectroscopy of DNA Hairpins (I): Irreversibility and Dissipation, J. Stat. Mech. (2009) P02060.

[31] F. E. Müllner, S. Syed, P. R. Selvin, and F. J. Sigworth, Improved Hidden Markov Models for Molecular Motors, Part 1: Basic Theory, Biophys. J. 99, 3684 (2010).

[32] J. M. Huguet, C. V. Bizarro, N. Forns, S. B. Smith, C. Bustamante, and F. Ritort, Single-Molecule Derivation of Salt Dependent Base-Pair Free Energies in DNA, Proc. Natl. Acad. Sci. U.S.A. 107, 15431 (2010).

[33] J. M. Huguet, N. Forns, and F. Ritort, Statistical Properties of Metastable Intermediates in DNA Unzipping, Phys. Rev. Lett. 103, 248106 (2009). 